\title{
ADAPTAÇÃO DE UMA ESCALA MULTIDIMENSIONAL DE IDENTIFICAÇÃO PARA PORTUGUÊS ${ }^{1}$
}

\author{
Miguel Ribeiro Ramos ${ }^{2}$ \\ Hélder Alves ${ }^{3}$
}

Resumo: Recentemente evoluiu-se de modelos unidimensionais do conceito de identificação grupal para concepções multidimensionais. Apesar destes avanços teóricos, a investigação em Portugal continua a usar escalas unidimensionais de identificação, o que dificulta a comparação de resultados entre estudos e não permite captar a complexidade deste conceito. Neste artigo apresentamos uma adaptação da escala multidimensional de identificação de Leach et al. (2008) para português. Os resultados mostraram uma medida fidedigna e com a estrutura factorial consistente com a de Leach et al. (2008), distinguindo os componentes satisfação, centralidade, solidariedade, auto-estereotipização e homogeneidade do endogrupo. A discussão centra-se sobre as vantagens do uso de uma perspectiva multidimensional.

Palavras-chave: identificação social, teoria da identidade social, medição; escala multidimensional

Portuguese adaptation of a multidimensional scale of identification (Abstract): The early unidimensional perspective of social identification has recently evolved to a multidimensional conceptualisation. Despite these theoretical advances, research conducted in Portugal still uses measures that reflect a unidimensional perspective of identification, which impedes an adequate comparison of results across studies and contributes to a misunderstanding of this construct. In the present article we provide a Portuguese adaptation of the multidimensional measure of Leach et al. (2008). Our results suggest a reliable measure with a factorial structure identical to Leach et al.'s (2008) model, in which we find the components of satisfaction, centrality, solidarity, self-stereotyping, and ingroup homogeneity. The discussion focuses on the advantages of using a multidimensional perspective.

${ }^{1}$ Esta investigação foi financiada pela Fundação para a Ciência e Tecnologia (PTDC/PSI-PSO/098110/2008)

2 CIS-IUL. Correspondência em relação a este artigo deve ser enviada para Miguel R. Ramos, CIS-IUL, Av. das Forças Armadas, 1649-026 Lisboa, Portugal. Email: mrrst@iscte.pt

3 CIS-IUL; ISCTE-IUL 
Keywords: Group identification, Social identity theory, measurement, multidimensional scale.

\section{Adaptação de uma escala multidimensional de identificação para português}

Desde a teoria de identificação social (Tafjel, 1978) que a temática da identificação grupal tem ocupado um lugar importante em psicologia social. De facto, os vários grupos a que pertencemos têm um impacto fundamental na forma como nos comportamos e posicionamos no meio social (Tajfel \& Turner, 1979; Turner, Hogg, Oakes, Reicher, \& Wetherell, 1987). Apesar do enorme avanço científico nesta área (ver Ellemers, Spears, \& Doosje, 1999), a forma como a identificação social é conceptualizada e medida tem sido alvo de debate e discórdia entre investigadores (Ashmoore, Deaux \& McLaughlin-Volpe, 2004).

Numa tentativa de uniformizar e criar novos padrões para a medição da identificação grupal, Leach e colaboradores (2008) analisaram múltiplas escalas de identificação social e definiram uma medida bidimensional. $\mathrm{O}$ trabalho de Leach et al. (2008) criou um novo padrão na medição de identificação grupal que tem sido usado em diversos estudos (e.g., Miron, Branscombe, \& Biernat, 2010; Nadler, Harpaz-Gorodeisky, \& Bem-David, 2009). Apesar destes avanços teóricos recentes, o trabalho de investigação em português tem recorrido a escalas unidimensionais que abordam diferentes aspectos de identificação (e.g., Ferreira \& Marques, 2007; Pimentel, Gouveia, \& Fonseca, 2005). Assim, não se verifica uma uniformização da medição do conceito de identificação grupal, impossibilitando muitas vezes comparações entre estudos e uma compreensão total dos resultados. Tendo em conta estas limitações, no presente artigo propomo-nos a introduzir uma adaptação da escala de Leach et al. (2008) à lingua Portuguesa (versão europeia), pois acreditamos que tal irá permitir uma maior uniformização do conceito, possibilitar a comparação de resultados entre estudos, e servir também o interesse de investigadores que pretendem analisar aspectos específicos da identificação grupal.

\section{Uma perspectiva multidimensional sobre a identificação social}

Uma questão importante para a investigação em psicologia social tem sido se a identificação social tem apenas uma ou várias dimensões separadas (Brown, Condor, Mathews, Wade, \& Williams, 1986; Ellemers, Kortekaas 
\& Ouwerker, 1999; Hinkle, Taylor Fox-Cardamone, \& Crook, 1989). Os modelos multidimensionais defendem que nenhuma dimensão de identificação social isolada é representativa desta mesma identificação. De facto, dimensões diferentes estão associadas a resultados e efeitos diferentes (Sellers et al., 1998). Por exemplo, num estudo com Afro-Americanos, Rowley, Sellers, Chavous e Smith (1998) verificaram que componentes afectivos da identificação estão positivamente relacionados com a auto-estima pessoal, quando estes indivíduos consideram o seu grupo étnico relevante para si. Noutro estudo, Spencer-Rodgers e Collins (2006) demonstraram que uma vinculação com o grupo mediou a relação entre as percepções de desvantagem do endogrupo e a auto-estima ${ }^{4}$. Em suma, a investigação tem sugerido que as identidades grupais têm efeitos diferentes dependendo nas dimensões que são analisadas (Jackson \& Smith, 1999).

O trabalho pioneiro sobre a medição de identificação social centrou-se no facto de que uma dada pertença grupal tem significados diferentes para indivíduos diferentes (Brown \& Williams, 1984). Recentemente, a investigação tem-se centrado no facto de que, dependendo da dimensão, uma dada pertença grupal pode ter significados diferentes para o mesmo indivíduo (Cameron, 2004; Cameron \& Lalonde, 2001; Ellemers et al., 1999; Jackson, 2002). Por exemplo, uma pessoa pode definir-se como Português enquanto sendo a sua identidade, mas pode sentir que não tem nada em comum com outros Portugueses, ou pode mesmo não estar contente por pertencer a este grupo.

A definição de Tajfel (1978) de identificação social permite ter uma visão mais detalhada sobre as dimensões envolvidas neste conceito. Para este autor, a identificação social é "a parte do auto-conceito que tem origem no seu conhecimento sobre a pertença a um dado grupo social (ou grupos) conjuntamente com o valor e significado emocional relacionado com este grupo" (Tajfel, 1978, p. 63, tradução nossa). A partir desta definição é possível distinguir três componentes da identificação social: um componente cognitivo (estar sensibilizado para uma pertença grupal); um componente avaliativo (a estima pelo grupo); e um componente emocional (aspectos emocionais de vinculação com o grupo) (Ellemers et al., 1999).

Apesar de haver algum consenso sobre a existência de uma multi-dimensionalidade da identificação social, não há um acordo quanto à natureza e número de dimensões. Por exemplo, Karasawa (1991) sugeriu que identificação com o grupo e identificação com os membros do grupo são

\footnotetext{
${ }^{4}$ Nos diversos estudos há uma grande divergência do nome atribuído às dimensões. Por exemplo, para Rowley et al. (1998) "vinculação com o grupo" é equivalente à "ligação com o endogrupo" de Cameron (2004) e, também, equivalente à "solidariedade" de Leach et al. (2008).
} 
aspectos diferentes da identificação social. Ellemers et al. (1999) argumentaram que a identificação social é composta por três factores: a auto-estima do grupo, a auto-categorização, e o empenho pelo grupo. Jackson (2002) também considerou três aspectos diferentes: a auto-categorização, uma avaliação emocional do grupo, e percepções emocionais de solidariedade para com o grupo. Cameron (2004) comparou medidas já validadas de identificação social que foram testadas em vários grupos. Desta análise emergiram três componentes: o cognitivo, o afectivo, e um de vinculação.

Mais recentemente, Leach et al. (2008) propuseram um modelo hierárquico de duas dimensões de identificação social, tendo sugerido as dimensões de auto-definição e de auto-investimento, ambas compostas por diversos factores que apresentamos nas próximas secções.

\section{Auto-definição: auto-estereotipização e homogeneidade do endogrupo}

A identificação com um determinado grupo é muito mais do que uma simples inclusão no grupo (Tajfel, 1978) e a auto-definição indica a forma como um indíviduo se define num nível grupal. Investigação anterior sugeriu que indivíduos que se identificam com um grupo tendem a percepcionar-se como sendo semelhantes a membros prototípicos do mesmo grupo (Oakes, Haslam, \& Turner, 1994). Leach et al. (2008) denominaram este processo de auto-estereotipização o qual está relacionado com uma despersonalização e inclusão no grupo. A auto-estereotipização é o grau em que um indivíduo se vê como semelhante e, também, com algo em comum em relação ao membro habitual do grupo. Portanto, uma pessoa deixa de se percepcionar a si própria enquanto indivíduo isolado e passa a percepcionar-se como sendo semelhante ao indivíduo prototípico do grupo (Oakes et al., 1994). Por exemplo, alguns(mas) Portugueses(as) podem achar que são muito parecidos com a sua representação do(a) Português(a) ${ }^{5}$ habitual enquanto outros(as) podem não concordar com essa perspectiva.

A auto-definição envolve ainda a homogeneidade do grupo, ou seja, a percepção do grau de semelhança entre os indíviduos do endogrupo (Doosje, Ellemers, \& Spears, 1995). É esta percepção que confere uma identidade social coerente e estabelece o contraste entre o endogrupo e o exogrupo (Oakes et al., 1994). Por exemplo, uns indivíduos podem pensar que os Portugueses são muito parecidos entre si, enquanto outros podem achar que os Portugueses pouco têm em comum.

\footnotetext{
${ }^{5}$ Doravante referir-nos-emos meramente à forma masculina e singular de modo a evitar uma proliferação de parêntesis com morfemas. Ainda que reflectindo o comum "masculino universal" (Amâncio, 2003, p. 701), julgamos que, neste contexto, se justifica pelo ganho na facilidade de leitura.
} 


\section{Auto-investimento: Centralidade, satisfação e solidariedade}

O auto-investimento está relacionado com investimento pessoal depositado num grupo ao qual o indivíduo pertence. As pessoas pertencem a vários grupos na nossa sociedade, mas nem todos são importantes num determinado tempo específico, ou seja, os vários grupos não têm sempre um significado psicológico equivalente (Deaux, Reid, Mizrahi, \& Ethier, 1995). Por um lado, o contexto tem um papel determinante em tornar uma dada identidade grupal saliente. Por outro lado, alguns indivíduos estão consistentemente mais inclinados para actuarem de acordo com uma determinada identidade em detrimento de outras identidades grupais (Gurin \& Markus, 1989; Oakes, 1987). Por exemplo, um emigrante português na Alemanha pode estar mais inclinado a comportar-se de acordo com a sua identidade nacional do que outros compatriotas na Alemanha. Neste caso, espera-se que a identidade nacional seja mais central para o primeiro indivíduo do que para os outros. Leach et al. (2008) definiram centralidade como sendo o grau de importância de uma dada identidade grupal para o auto-conceito do indivíduo. Mais especificamente, trata-se a frequência com que uma identidade grupal específica se torna activa em contextos diferentes. Outros autores definiram este conceito de uma forma semelhante, argumentando que a centralidade está associada a uma importância subjectiva de uma dada identidade grupal para o self (Hutnik, 1991; Rosenberg, 1979; Sellers et al., 1997).

Uma segunda dimensão de auto-investimento está relacionada com um componente afectivo que se encontra presente na maior parte das escalas de identificação grupal (e.g., Brown et al., 1986; Ellemers et al., 1999), por exemplo, em escalas de identificação étnica (e.g., Phinney, 1992) e racial (e.g., Sellers et al., 1997). Leach et al. (2008) definiram este componente como satisfação, reflectindo uma avaliação sobre a identidade grupal e relacionado com as emoções específicas que emergem de uma pertença grupal específica. Por exemplo, alguns Portugueses podem gostar e sentir-se bem em ser Portugueses, enquanto outros podem ter avaliações negativas sobre o grupo nacional a que pertencem.

Por fim, é importante também considerar os laços que as pessoas desenvolvem em relação aos outros membros do mesmo grupo. Estes laços dão um sentido de pertença e trazem proximidade emocional (Cameron, 2004). A solidariedade está relacionada com a percepção de pertença a um grupo e com os laços psicológicos que ligam o self ao grupo (Cameron, 2004). De uma forma idêntica, outros autores conceptualizaram a solidariedade como sendo um vínculo entre a pessoa e os membros do grupo (Cameron \& Lalonde, 2001), ou um sentimento de pertença ao grupo (Phinney, 1992). Por exemplo, alguns Portugueses podem sentir-se ligados aos outros 
Portugueses enquanto outros podem sentir-se desprendidos ou separados dos membros desse grupo nacional.

\section{O presente trabalho}

A nossa investigação centrou-se no modelo bidimensional de Leach et al. (2008), por várias razões. Primeiro, como inicialmente argumentámos, apesar do elevado suporte empírico a favor de diversas dimensões de identificação social (e.g., Ellemers et al., 1999; Jackson, 2002; Sellers et al., 1998), não há consenso sobre a natureza e número de dimensões. Em relação à medição de identificação social, as múltiplas metodologias usadas em estudos anteriores não permitem compreender a diversidade dos seus factores estruturais. A escala de identificação social de Leach et al. (2008) é baseada em múltiplas análises factoriais de identificação, tendo sido testadas escalas como as de identificação social de Brown et al. (1986) e de Hinkle et al. (1989), de auto-estima colectiva (Luhtanen \& Crocker, 1992), assim como trabalho envolvendo a identificação com as mulheres (Gurin \& Markus, 1989; Gurin \& Townsend, 1986). Este modelo foi sujeito a uma análise de modelos estruturais, permitindo comparar um modelo bidimensional com outros tridimensionais ou unidimensionais. Os resultados indicaram que um modelo bidimensional fornece uma explicação mais adequada aos dados do que as outras alternativas. As dimensões que emergiram desta análise estão na mesma linha de investigação anterior que tinha distinguido um componente cognitivo, um afectivo, e outro de vinculação para com o grupo (e.g., Boatswain \& Lalonde, 2000; Cameron, 2004).

Um importante segundo aspecto prende-se com o facto de esta escala ter sido testada com várias categorias sociais (e.g., estudante universitário, nacionalidade, grupo de cidadãos Europeus). Assim, em vez de ser uma escala específica para um determinado grupo, esta permite ser adaptada para vários tipos de grupos, incluindo grandes categorias sociais como nacionalidade e etnicidade.

Uma terceira razão relaciona-se com o facto de este modelo ter sido testado com variáveis adicionais tais como medidas de personalidade e outras específicas para grupos, permitindo uma avaliação das validades convergente e discriminante. Os resultados demonstraram que os vários componentes estão consistentemente associados a outras variáveis. Por exemplo, a necessidade de pertencer a grupos sociais está unicamente associada com a solidariedade, enquanto a auto-estima colectiva está unicamente relacionada com a satisfação (Leach et al., 2008). 


\section{Método}

\section{Participantes e Procedimento}

Participaram neste estudo 478 estudantes universitários. A amostra foi composta por 183 pessoas do sexo masculino e 295 do sexo feminino. A sua idade variou entre os 17 e os 60 anos $(M=23.28 ; D P=7.24 ; M d=20$; Moda $=19)$. Neste estudo medimos a identificação com uma universidade em Lisboa (por motivos de anonimato denominámos de universidade $\mathrm{A})^{6}$.

Aplicámos uma versão traduzida para Português da escala de Leach et al. (2008) cujos itens podem ser consultados em Anexo. Os participantes responderam em escalas entre 1 "discordo completamente" e 7 "concordo completamente", sendo que um valor mais alto indica um maior grau de identificação.

\section{Resultados}

\section{Análise descritiva e fidelidade}

A Tabela 1 indica os alfas de Cronbach, médias, desvios-padrão e correlações entre as variáveis. Em relação à fidelidade, uma análise dos alfas de Cronbach indica que as várias subescalas tiveram uma elevada consistência interna, variando entre .76 (centralidade) e .92 (auto-estereotipização). Em relação às médias, a satisfação foi o componente com valores mais altos $(M$ $=5.84$ ), enquanto a auto-estereotipização foi o que teve um valor mais baixo $(M=3.96)$. Tal como no modelo original, as correlações entre as subescalas são positivas e significativas (valores entre .27 e .57 ; $p s<.01$ ).

\section{Análise factorial confirmatória}

Tal como consta na Figura 1, para testarmos a nossa adaptação seguimos o mesmo procedimento de Leach et al. (2008) e testámos, através de uma análise factorial confirmatória, o modelo A numa primeira fase, e numa segunda fase comparámos este modelo com vários modelos alternativos propostos pelos autores da escala (modelos B a F). Para este efeito recorremos ao software AMOS versão 17.

\footnotetext{
${ }^{6} \mathrm{O}$ estudo aqui apresentado reúne os dados de diversos estudos em que a escala foi aplicada como parte de questionários diferentes. Estes questionários tinham como objectivo abordar questões relacionadas com a identificação e percepções de justiça. Agregámos os dados porque as amostras pertencem à mesma população e em todas foi medida a mesma pertença grupal.
} 
Tabela 1. Coeficientes de fidelidade, médias, desvios-padrão e correlações das dimensões de identificação.

\begin{tabular}{lccccccc}
\hline Dimensão & $\alpha$ & $M(D P)$ & 1 & 2 & 3 & 4 & 5 \\
\hline 1. Auto-ester. & .92 & $3.96(1.50)$ & - & & & & \\
2. Homog. endogrupo & .82 & $4.18(1.36)$ & $.46^{*}$ & - & & & \\
3. Satisfação & .87 & $5.84(1.01)$ & $.45^{*}$ & $.27^{*}$ & - & & \\
4. Solidariedade & .84 & $4.36(1.33)$ & $.46^{*}$ & $.38^{*}$ & $.51^{*}$ & - & \\
5. Centralidade & .76 & $4.91(1.35)$ & $.44^{*}$ & $.35^{*}$ & $.57^{*}$ & $.52^{*}$ & - \\
\hline
\end{tabular}

Nota: $* p<.01$

Figura 1. O modelo proposto por Leachet al. (2008; A) e os restantes modelos alternativos também propostos pelos autores (adaptado de Leachet al., 2008).

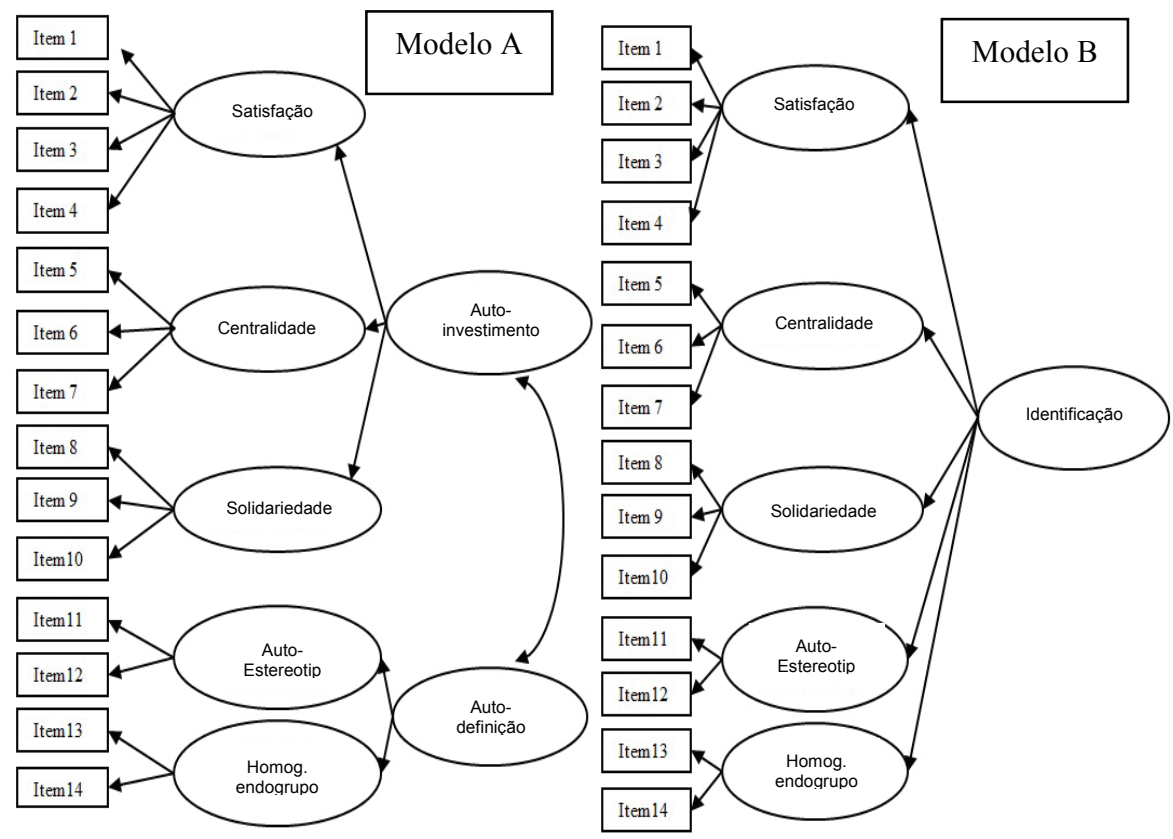



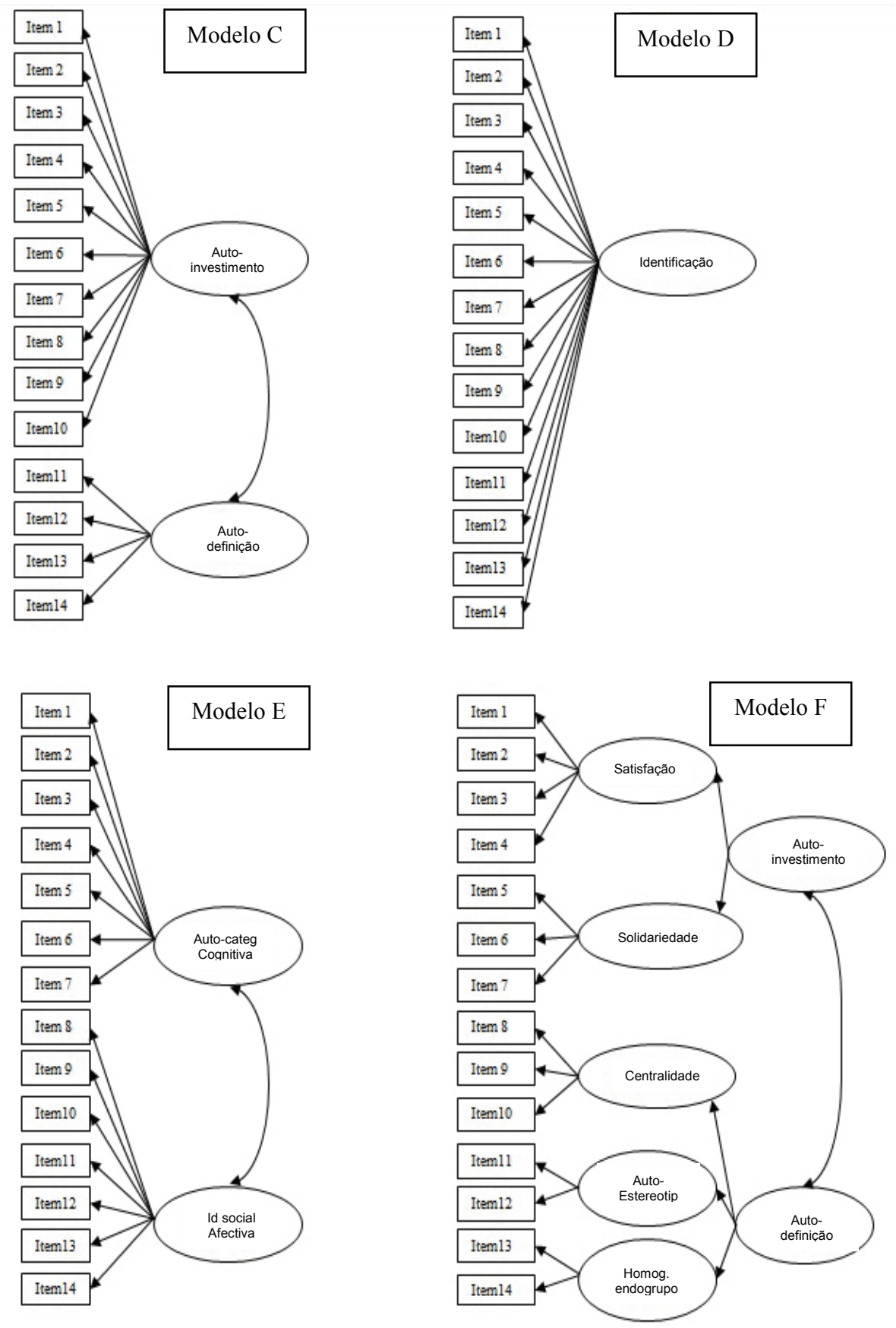
Como pode ser consultado na Tabela 2, s resultados da análise factorial confirmatória indicam que para o modelo A os índices de ajustamento do modelo (CFI e NFI) são satisfatórios. Ambos os valores estão acima do mínimo recomendado de .95 . O índice de resíduos principais (RMSEA) situa-se abaixo do valor máximo recomendado de .08 (ver Hu \& Bentler, 1999). A Figura 2 indica os valores estandardizados dos pesos factoriais obtidos para o modelo A. Como se pode observar, todos os factores estão fortemente relacionados com os itens correspondentes, apresentando pesos factoriais entre .43 e .96 (todos os $p s<.001$ ). Os pesos factoriais entre as dimensões de primeira e segunda ordem apresentam pesos factoriais elevados (acima de .62 e $p<.001$ ). Por fim, as duas dimensões de segunda ordem estão correlacionadas $(.83 ; p<.001)$.

Figura 2. O modelo A e o peso factorial dos diversos itens.

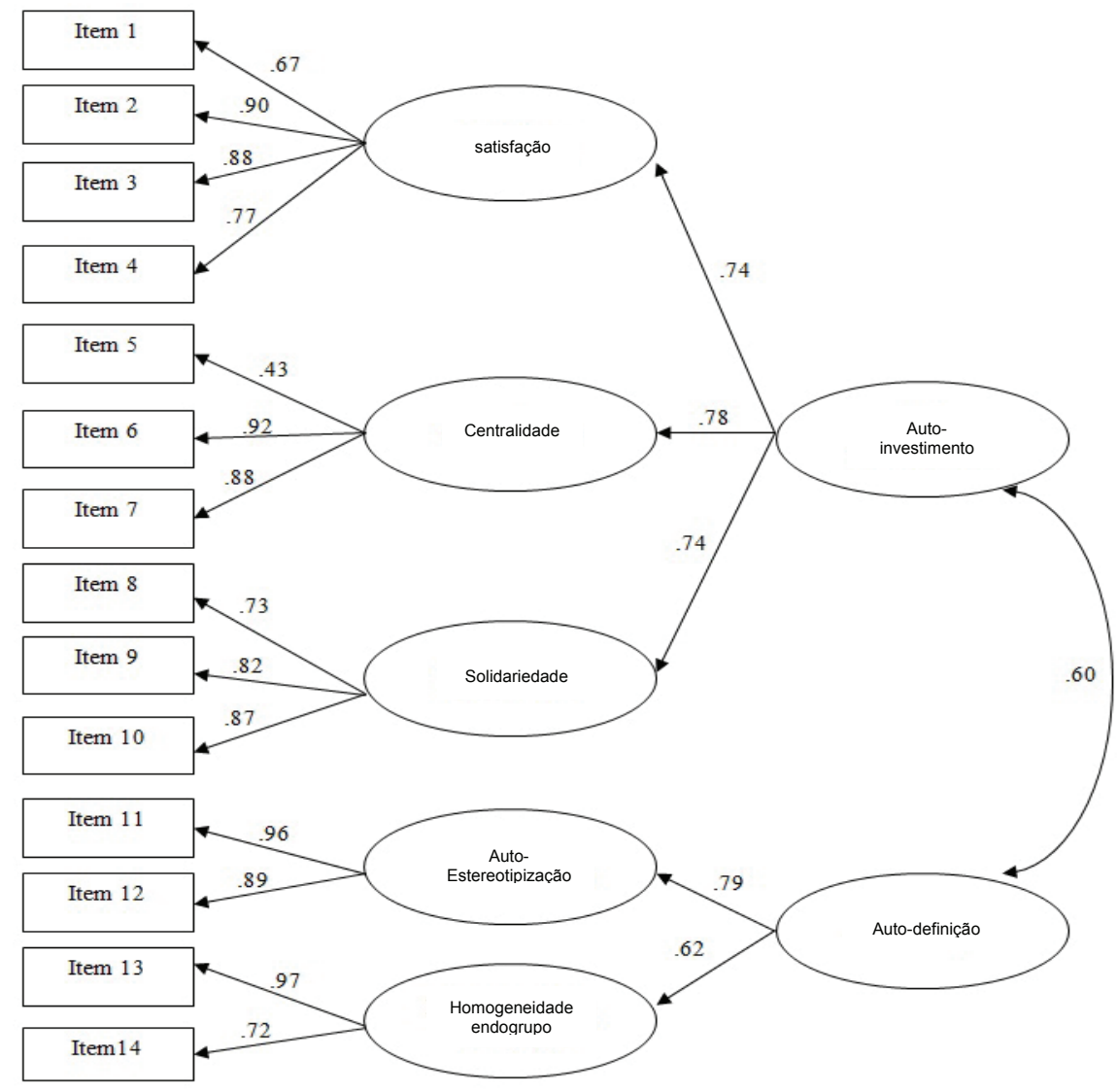


Tabela 2. Comparação dos diferentes modelos.

\begin{tabular}{ccccccc}
\hline Modelo & CFI & NFI & RMSEA & AIC & $\mathrm{x}^{2}$ & $\mathrm{gl}$ \\
\hline $\mathrm{A}$ & .95 & .93 & .076 & 362.209 & $266.21^{*}$ & 71 \\
$\mathrm{~B}$ & .95 & .93 & .078 & 375.546 & $281.55^{*}$ & 72 \\
$\mathrm{C}$ & .71 & .69 & .179 & 1319.368 & $1233.37^{*}$ & 76 \\
$\mathrm{D}$ & .59 & .58 & .210 & 1779.548 & $1695.55^{*}$ & 76 \\
$\mathrm{E}$ & .54 & .53 & .223 & 1984.757 & $1900.76^{*}$ & 77 \\
$\mathrm{~F}$ & .95 & .93 & .079 & 375.967 & $279.967^{*}$ & 71 \\
\hline
\end{tabular}

Nota: $* p<.001$

Numa segunda fase, comparámos o modelo A com os modelos alternativos. Como indica a Tabela 2 , os únicos modelos com índices de ajustamento satisfatório são os modelos A, B, e F (valores de CFI e NFI acima de .95 e de RMSEA abaixo de .08). Estes três modelos têm em comum uma estrutura com cinco factores. No entanto, como os modelos de $\mathrm{A}$ a $\mathrm{F}$ não são nested models $^{7}$, recorremos aos valores de AIC (Akaike Information Criterion) para compararmos o seu ajustamento aos dados (ver Kline, 1988). Os resultados mostram que o modelo A é o que apresenta o valor mais baixo $(\mathrm{AIC}=362.209)$ e, portanto, o modelo que melhor explica a matriz de covariâncias. Desta forma, os nossos resultados sugerem que o modelo de Leach et al. (2008) foi também na nossa tradução o modelo mais adequado mostrando cinco factores (i.e., solidariedade, centralidade, satisfação, auto-estereotipização, e homogeneidade do endogrupo) agrupados em duas dimensões (i.e., auto-investimento e auto-definição).

\section{Discussão}

O trabalho de investigação realizado em Portugal não tem, de acordo com o nosso conhecimento, acompanhado um desenvolvimento de uma perspectiva unidimensional da identificação para uma multidimensional. De

\footnotetext{
${ }^{7}$ Dois modelos são considerados nested quando o segundo se obtém através da modificação de parâmetros do primeiro (por exemplo, restringir um parâmetro do modelo inicial a zero para obter um segundo modelo). Os modelos que testámos não são nested porque não só os parâmetros variam como as variáveis também.
} 
forma a colmatar esta lacuna, a presente investigação teve o objectivo de adaptar uma escala multidimensional de identificação - a de Leach et al. (2008) - para a língua portuguesa.

Os resultados obtidos no nosso estudo foram consistentes com os de Leach et al. (2008). A nossa análise dos itens traduzidos para português confirmou a estrutura factorial original. Assim, identificámos os cinco componentes da escala: auto-estereotipização, homogeneidade do grupo, satisfação, centralidade e solidariedade; agrupados nas dimensões de auto-investimento e auto-definição. Estes resultados foram acompanhados por coeficientes de fidelidade elevados para os componentes, assim como correlações altas entre os itens. Importante também, foi o facto de quando compararmos o modelo de Leach et al. (2008) com outros modelos alternativos, o modelo sugerido pelos autores ter sido aquele que apresentou melhor ajustamento aos dados.

A nossa tradução oferece pelo menos três vantagens em relação à actual literatura para os investigadores que pretendem conduzir estudos em português. Primeiro, ao identificar cinco componentes e ao apresentar uma definição mais específica de identificação social, contribui-se para uma maior uniformização do próprio conceito. De facto, entre vários estudos a identificação é medida frequentemente com significados diferentes. Por exemplo, estudos que recorrem à escala de Luhtanen e Crocker (1992) medem uma satisfação com o grupo, enquanto que aqueles que recorrem à escala de Gurin e Markus (1989) medem aspectos de centralidade. Portanto, torna-se difícil compreender o alcance dos resultados e mesmo comparar estes resultados entre estudos. É importante, então, usar uma medida que seja consensual e ofereça uma definição pormenorizada do conceito de identificação social.

Segundo, alguma investigação recente tem apontado para o facto de que os diversos componentes de identificação social têm um papel diferente quando analisados em processos intergrupais. Estes processos incluem respostas à discriminação (Ramos, Cassidy, Reicher, \& Haslam, no prelo), coping em situações de estigmatização (Crabtree, Haslam, Postmes, \& Haslam, 2010), e ajustamento psicológico (Leach, Mosquera, Vliek, \& Hirt, 2010). Estes resultados devem encorajar os investigadores a testar novos modelos teóricos e também a formular hipóteses mais específicas, enquadrando os diversos componentes de identificação social.

Por fim, nos nossos estudos medimos a identificação com um grupo real que é relativamente abrangente (ser aluno de uma universidade). De certa forma, os resultados sugerem que os itens apresentados neste artigo poderão ser adaptados para uma série de grupos reais, incluindo importantes categorias sociais tais como, nacionalidade, e etnia, embora não os tenhamos abordado aqui.

Apesar das vantagens apontadas, há duas limitações do nosso estudo que não devem ser negligenciadas. A primeira limitação prende-se com o 
facto de que os participantes foram todos estudantes universitários e de uma faixa etária relativamente jovem. Uma segunda limitação é a de que a escala de identificação foi inserida em estudos com outros objectivos e variáveis que podem ter afectado as respostas. Apesar destas duas limitações, os nossos resultados foram consistentes e replicaram a estrutura factorial original que tinha sido demonstrada com grupos etários mais abrangentes e estudos com paradigmas diferentes.

\section{Conclusão}

Apresentámos neste estudo uma versão traduzida para português da escala de Leach et al. (2008). Os nossos resultados foram consistentes com os dos autores da escala permitindo uma validação para o contexto de investigação em português. Acreditamos que este contributo possa ter importantes implicações não só teóricas mas também na forma como são colocados os problemas de investigação. Por fim, acreditamos, também, que a nossa investigação possa contribuir para um maior foco sobre o que significa a identificação social e qual o significado que esta tem para os indivíduos num contexto intergrupal.

\section{Referências}

Amâncio, L. (2003). O género no discurso das ciências sociais. Análise Social, XXXVIII, 687-714.

Ashmore, R. D., Deaux, K., \& McLaughlin-Volpe, T. (2004). An organizing framework for collective identity: Articulation and significance of multidimensionality. Psychological Bulletin, 130, 80-114.

Boatswain, S. J., \& Lalonde, R. N. (2000). Social identity and preferred ethnic/racial labels for Blacks in Canada. Journal of Black Psychology, 26, 216-234.

Brown, R., Condor, S., Mathews, A., Wade, G., \& Williams, J. (1986). Explaining intergroup differentiation in an industrial organization. Journal of Occupational Psychology, 59, 273-286.

Brown, R., \& Williams, J. (1984). Group identification: The same thing to all people? Human Relations, 37, 547-564.

Cameron, J.E. (2004). A three-factor model of social identity. Self and Identity, 3, 239-262.

Cameron, J. E., \& Lalonde, R. N. (2001). Social identification and gender-related ideology in women and men. British Journal of Social Psychology, 40, 59-77.

Crabtree, J., Haslam, S. A., Postmes, T. \& Haslam, C. (2010). Mental health support groups, stigma and self-esteem: Positive and negative implications of group identification. Journal of Social Issues, 66, 556-569. 
Deaux, K., Reid, A., Mizrahi, K., \& Cotting, D. (1999). Connecting the person to the social: The functions of social identification. In T. R. Tyler, R. M. Kramer, \& O. P. John (Eds.), The psychology of the self (pp. 91-113). Hillsdale, NJ: Lawrence Erlbaum Associates, Inc.

Doosje, B., Spears, R., \& Ellemers, N. (1995). Perceived intragroup variability as a function of group status and identification. Journal of Experimental Social Psychology, 31, 410-436.

Ellemers, N., Kortekaas, P., \& Ouwerker, J. W. (1999). Self-categorisation, commitment to the group and group self-esteem as related but distinct aspects. $E u$ ropean Journal of Social Psychology, 29, 371-389.

Ellemers, N., Spears, R., \& Doosje, B. (1997). Social identity: Context, commitment, content. Oxford: Blackwell.

Ferreira, P. M. (2007). Assimetrias de poder intergrupal e percepção de desvio intragrupal. Tese de doutoramento não publicada. Universidade do Porto.

Gurin, P., \& Markus, H. (1989). Cognitive consequences of gender identity. In S. Skevington \& D. Baker (Eds.), The social identity of women (pp. 152-172). London: Sage.

Gurin, P., \& Townsend, A. (1986). Properties of gender identity and their implications for gender consciousness. British Journal of Social Psychology, 25, 139-148 .

Hinkle, S. Taylor, L.A., Fox-Cardamone, D. L., \& Crook, K. F. (1989). Intragroup identification and intergroup differentiation: A multicomponent approach. British Journal of Social Psychology, 28, 305-317.

Hutnik, N. (1991). Ethnic minority identity: A social psychological perspective. Oxford: Clarendon Press.

Jackson, J. W. (2002). Intergroup attitudes as a function of different dimensions of group identification and perceived intergroup conflict. Self and Identity, 1, 11-33 .

Jackson, J. W., \& Smith, E. R. (1999). Conceptualizing social identity: A new framework and evidence for the impact of different dimensions. Personality and Social Psychology Bulletin, 25, 120-135.

Karasawa, M. (1991). Toward an assessment of social identity: The structure of group identification and its effects on in-group evaluations. British Journal of Social Psychology, 30, 293-307.

Leach, C. W., Mosquera, P. R., Vliek, M., \& Hirt, E. (2010). Group devaluation and group identification. Journal of Social Issues, 66, 535-552.

Leach, C. W., van Zomeren, M., Zebel, S., Vliek, M. L., Pennekamp, S. F., Doosje, B., Ouwerkerk, J. W., \& Spears, R. (2008). Group-level self-definition and self-investment: a hierarchical (multicomponent) model of ingroup identification. Journal of Personality and Social Psychology, 95, 144-165.

Luhtanen, R., \& Crocker, J. (1992). A Collective Self-esteem Scale: Self-evaluation of one's social identity. Personality and Social Psychology Bulletin, 18, 302-318 .

Miron, A. Branscombe, N. \& Biernat, M. (2010). Motivated shifting of justice standards. Personality and Social Psychology Bulletin, 36, 768-779. 
Nadler, A., Harpaz-Gorodeisky, G., \& Ben-David, Y. (2009). Defensive helping: Threat to group identity, ingroup identification, status stability, and common group identity as determinants of intergroup help-giving. Journal of Personality and Social Psychology, 97, 823-834.

Oakes, P. J. (1987). The salience of social categories. In J. C. Turner (Ed.), Rediscovering the social group: A self-categorization theory (pp. 117-141). Oxford: Basil Blackwell.

Oakes, P., Haslam, S. A., \& Turner, J. C. (1994). Stereotyping and social reality. Oxford: Blackwell.

Phinney, J. S. (1992). The Multigroup Ethnic Identity Measure: A new scale for use with diverse groups. Journal of Adolescent Research, 7, 156-176.

Pimentel, C. E., Gouveia, V. V., \& Fonseca P. N. (2005). Escala de identificação com grupos alternativos: construção e comprovação da estrutura factorial. PsicoUSF, 10, 121-127.

Ramos, M. R., Cassidy, C., Reicher, S., \& Haslam, S. A. (no prelo). A longitudinal investigation of the rejection-identification hypothesis. British Journal of Social Psychology.

Rosenberg, R. (1979). Conceiving the self. New York: Basic Books.

Rowley, S. J., Sellers, R. M., Chavous, T. M., \& Smith, M. A. (1998). The relationship between racial identity and self-esteem in African-American college and high school students. Journal of Personality and Social Psychology, 74, 715-724 .

Sellers, R. M., Smith, M. A., Sheltone, S. A. J., Rowley, T. M., \& Chavous, T. M. (1998). Multidimensional model of racial identity: A reconceptualization of African American racial identity. Personality and Social Psychology Review, 2(1), 18-39.

Sellers, R. M., Rowley, S. A. J., Chavous, T. M., Shelton, J. N., \& Smith, M. A. (1997). Multidimensional Inventory of Black Identity: A preliminary investigation of reliability and construct validity. Journal of Personality and Social Psychology, 73, 805-815.

Spencer-Rodgers, J., \& Collins, N. L. (2006). Risk and resilience: Dual effects of perceptions of group disadvantage among Latinos. Journal of Experimental Social Psychology, 42, 729-737.

Tabachnik, B., \& Fidell, L. S. (2001). Using multivariate statistics. Needham Heights: Allyn \& Bacon.

Tajfel, H. (1978). The social psychology of minorities. London: Minority Rights Group.

Tajfel, H., \& Turner, J.C. (1979). An integrative theory of intergroup conflict. In W.G. Austin \& S. Worchel (Eds.) The social psychology of intergroup relations (pp. 33-47). Monterey, California: Brooks/Cole.

Turner, J. C., Hogg, M. A., Oakes, P. J., Reicher, S. D., \& Wetherell, M. (1987). Rediscovering the social group: A self-categorization theory. Oxford: Blackwell. 


\footnotetext{
Anexo

1 - Eu penso que os [membros do endogrupo] têm muito de que se orgulhar.

2 - É agradável ser um [membros do endogrupo].

3 - Ser um [membros do endogrupo] dá-me uma sensação agradável.

4 - Eu estou contente por ser um [membros do endogrupo].

5 - Eu penso muitas vezes no facto de que sou um [membros do endogrupo].

6 - O facto de que sou um [membros do endogrupo] é uma parte importante da minha identidade.

7 - Ser um [membros do endogrupo] é uma parte importante de como eu me vejo a mim mesmo.

8 - Eu sinto uma ligação com os [membros do endogrupo].

9 - Eu sinto solidariedade para com os [membros do endogrupo].

10 - Eu sinto dedicação para com os [membros do endogrupo].

11 - Eu tenho muito em comum com o habitual [membros do endogrupo].

12 - Eu sou parecido com o habitual [membros do endogrupo].

13 - Os [membros do endogrupo] têm muitos pontos em comum entre si.

14 - Os [membros do endogrupo] são muito parecidos.
} 